\title{
Lamotrigin - neue Sicherheitssignale bei Einnahme in der Schwangerschaft
}

Tobias Leniger
Die Firma GlaxoSmithKline AG hat Swissmedic neue Erkenntnisse zu teratogenen Effekten unter Einnahme von Lamotrigin $\left(\right.$ Lamictal ${ }^{\circledR}$ ) während der Schwangerschaft mitgeteilt.

Zum einen wurde eine deutliche, dosisabhängige Zunahme von «major congenital malformations» (MCM) bei Tagesdosen von $\geq 200 \mathrm{mg}$ gefunden [1]. Zum anderen wurde erstmals ein Signal für eine signifikant erhöhte Rate an Gaumenspaltbildungen unter Lamotrigin beobachtet. Hierbei handelt es sich um noch unpublizierte Daten aus einem preliminären Report der North American Antiepileptic Drug (NAAED) Pregnancy Registry. Die ausführlichen Daten sollen in naher Zukunft publiziert werden.

Weder die erhöhte Anzahl an Spaltbildungen noch eine Dosisabhängigkeit der Fehlbildungen konnten in anderen Datenbanken bisher bestätigt werden. Aufgrund der derzeitig begrenz- ten Datenlage ist aber zu vermuten, dass das Risiko von MCM für Neugeborene unter einer Exposition von Lamotrigin in der Schwangerschaft im Vergleich zu Neugeborenen ohne eine Exposition grenzwertig erhöht ist. Zu betonen ist aber auch, dass dieses Risiko scheinbar niedriger ist als z. B. für «ältere» Antikonvulsiva wie Phenytoin, Phenobarbital oder Valproinsäure.

In Absprache mit Swissmedic wird die Firma GlaxoSmithKline AG in den nächsten Wochen weitere Daten zur Verfügung stellen. Swissmedic wird dann eine der neuen Sicherheitslage entsprechende Veränderung der Fachinformation aller lamotriginhaltigen Präparate herbeiführen. Wie alle Antikonvulsiva sollte Lamotrigin nur bei klarer Indikation und in der niedrigst möglichen Dosis in der Schwangerschaft angewendet werden.

\section{Lamotrigine: nouvelles données sur les effets tératogènes en cas de prise pendant la grossesse}

1 Morrow J, Russell A, Guthrie E, Parsons L, Robertson I, Waddell R, et al. Malformation risks of antiepileptic drugs in pregnancy: a prospective study from the UK Epilepsy and Pregnancy Register. J Neurol Neurosurg Psychiatry 2006;77:193-8.

Korrespondenz/Correspondance: PD Dr. med. Tobias Leniger Swissmedic,

Schweizerisches Heilmittelinstitut Hallerstrasse 7

CH-3000 Bern 9

www.swissmedic.ch
La société GlaxoSmithKline SA a transmis à Swissmedic de nouvelles données sur les effets tératogènes liés à la prise de lamotrigine (Lamic$\operatorname{tal}^{\circledR}$ ) pendant la grossesse.

Ainsi, une nette augmentation dose-dépendante des malformations congénitales majeures (MCM) a été mise en évidence à des doses journalières $\geq 200 \mathrm{mg}$ [1]. De plus, les observations semblent indiquer pour la première fois une augmentation significative du taux de fentes palatines chez les enfants de mères traitées avec de la lamotrigine. Ces données encore non publiées proviennent d'un rapport préliminaire du «North American Antiepileptic Drug (NAAED) Pregnancy Registry». Mais elles devraient être prochainement publiées dans leur intégralité.

Jusqu'à présent, les autres banques de données existantes n'ont pu confirmer ni le plus grand nombre de fentes palatines ni une relation de dose-dépendance par rapport aux malformations. Mais, au vu des données partielles au- jourd'hui disponibles, il est probable que le risque de MCM chez les nouveau-nés exposés in utero à la lamotrigine soit légèrement supérieur à celui des nouveau-nés non exposés à ce produit. Mais il convient aussi de souligner que ce risque est apparemment inférieur à celui inhérent, par exemple, aux anticonvulsivants «plus anciens» tels que la phénytoïne, le phénobarbital ou l'acide valproïque.

D'entente avec Swissmedic, l'entreprise pharmaceutique GlaxoSmithKline SA communiquera ces prochaines semaines de nouvelles données. Swissmedic demandera alors à ce que l'information professionnelle de toutes les préparations à base de lamotrigine soit modifiée eu égard aux nouvelles connaissances sur les risques liés à ce principe actif. Enfin, à l'instar de tous les anticonvulsivants, la lamotrigine ne devrait être administrée pendant la grossesse que si l'indication est clairement établie et à la dose la plus faible possible. 